\title{
Editorial
}

\section{Are We Sleeping Enough?}

\author{
doi: http://dx.doi.org/10.3329/jemc.v7i3.34070
}

A good night sleep is essential to good health. Adequate sleep is necessary for restoration of energy, retention of memory and maintenance of our immune system. Internal circadian clock promotes sleep daily at night. There are two types of sleep: non-rapid eye movement (NREM) sleep and rapid eye movement (REM) sleep. NREM sleep occurs first and after a transitional period it is called slow-wave sleep or deep sleep. Body temperature and heart rate fall during this stage. Human secretes burst of growth hormone during deep sleep. REM sleep is of small duration and dreams are experienced in this phase. The sleep cycle of alternate NREM and REM sleep takes an average of 90 minutes, which occurs 4-6 times in a good night's sleep.

Sleep is considered adequate when there is no daytime sleepiness or dysfunction. Recommended period of sleep varies with age. According to National Sleep Foundation (NSF), American school age children (6-13 years) need 9-11 hours, teenagers (14-17 years) $8-10$ hours, younger adults (18-25 years) and adults (26-64 years) need 7-9 hours and older adults (65 years + ) need 7-8 hours. But practically, duration of sleep has reduced to a great extent in each group leading to development of a sleep-deprived society.

Academic burden has been increased in the schools and colleges in many countries including Bangladesh. Children, adolescents and young adults remain busy with their huge amount of studies and homework other than teaching periods. Dependency on the coaching centers and private tutorials reduced their leisure time and bed time. Striving for good grades, participation in extra-curricular activities, lack of rules about bedtimes and socializing are important factors which further interfere with duration of sleep. Moreover, with the advent of modern technology, television, personal computers, laptops, tablets, video games, smart phones etc keep them busy and make them late for going to sleep. According to the National Sleep Foundation's 2006 Sleep in America Poll, almost all adolescents had at least one media electronic device in their bedroom. ${ }^{2}$ Sleep research scientists reported that exposure to blue light suppresses melatonin which allows transition to sleep. ${ }^{3}$ Unhealthy sleep behavior, voluntary sleep restriction and irregular sleep-wake pattern reduce both duration and quality of sleep in these younger people. Chronic sleep loss has been linked to an increased risk of obesity, suicidal ideation, depression, drug and alcohol addiction.

Modern lifestyles are depriving adult people of adequate sleep. Rising demands in the workplace, increased working hours, poor work-life balance, social stress, high consumption of caffeine and nicotine, substance abuse etc are contributing to short sleep duration. According to a survey conducted by the US Centers for Disease Control (CDC) between 2005 and 2007 , more than $30 \%$ of adults slept less than six hours a night. ${ }^{4}$ Insufficient sleep is associated with an increased risk of developing inflammation, stress, anxiety, obesity, diabetes, hypertension, heart disease, stroke, depression and fatigue-related accidents. ${ }^{5}$ The CDC declared that insufficient sleep was becoming a public health epidemic. ${ }^{4}$

Sleep architecture or change in sleep pattern is a part of normal aging process. This may cause sleep problems in adults. Studies on the sleep habits of older Americans show an increase in the time to fall asleep (sleep latency), decline in REM sleep and an increase in sleep fragmentation (waking up during the night) with age. Prevalence of sleep disorders such as insomnia, sleep apnea, snoring, restless leg syndrome, periodic limb movement disorder etc increases with age. Studies also suggest that several physical and psychiatric illnesses and the medications contribute much to the development of sleep disturbances in the elderly people. ${ }^{6}$ 
Sleeping is considered as a leisure activity in society. But it plays a crucial role in maintaining good health. Chronic sleep deprivation especially in young adults and adults is a threat to physical and mental health, academic success and productivity of a nation. There are many physical and mental benefits of sleep. Though duration of sleep was mentioned in NSF recommendation, quality of sleep, rather than quantity should be considered. Keeping a regular schedule for sleep both in weekdays and weekends, turning off all electronic devices at least 15-20 minutes before bed, including a wind-down period at the end of the day might be helpful to get a good night sleep.

Healthcare providers should create awareness among general people about the importance of sleep. Advocating of educational, administrative and health policies for promoting healthy sleep and reducing the risk for sleep loss at local and national levels would help people of all age to lead a healthy and productive life.

\section{Rukhsana Parvin}

Associate Professor, Department of Medicine Enam Medical College \& Hospital, Savar, Dhaka Email: rukhsana_parvin@yahoo.com

\section{References}

1. National Sleep Foundation recommends new sleep times. Available at: https://sleepfoundation.org/pressrelease/national-sleep-foundation-recommends-newsleep-times. Accessed August 2017.

2. National Sleep Foundation. 2006 Teens and sleep. Sleep in America Polls. Washington, DC: National Sleep Foundation; 2006. Available at: www. sleepfoundation.org/article/sleep-america-polls/2006teens-and-sleep. Accessed August 2017.

3. Cain N, Gradisar M. Electronic media use and sleep in school-aged children and adolescents: a review. Sleep Med 2010; 11(8): 735-742.

4. Schoenborn CA, Adams PF. Health behaviors of adults: United States, 2005-2007. National Center for Health Statistics. Vital and Health Statistics. Series 10. Number 245.

5. Vgontzas AN, Mastorakos G, Bixler EO, Kales A, Gold PW, Chrousos GP. Sleep deprivation effects on the activity of the hypothalamic-pituitary-adrenal and growth axes: potential clinical implications. Clin Endocrinol (Oxf) 1999; 51(2): 205-215.

6. Franzen PL, Buysse DJ. Sleep disturbances and depression: risk relationships for subsequent depression and therapeutic implications. Dialogues Clin Neurosci 2008; 10(4): 473-481 